PREHOSPITAL CARE

\title{
Contemporary UK paramedical training and education. How do we train? How should we educate?
}

S Cooper

Correspondence to: Dr S Cooper, Faculty of Health and Social Work, C403, Portland Square, University of Plymouth, Plymouth, Devon PL4 8AA, UK; simon.cooper@ plymouth.ac.uk

Accepted

14 December 2004

Objective: To develop an understanding of the current system and future development of training and education within a large UK ambulance trust, based upon the experiences, beliefs, and opinions of stakeholders.

Methods: This was a qualitative naturalistic inquiry using an interpretative constructivist approach for 44 interviews with a range of ambulance staff. Stakeholder views on training and education were analysed and compared using the constant comparison method.

Results: Key emergent themes included: issues around prescribed programme entry levels and methods; the desire for a higher education curriculum with a balance between theory and practice; valid and reliable assessment methods; development of a supportive mentorship framework; an emphasis on self directed professional development with a focus on deskilling issues; and development of interprofessional collaborative links.

Conclusion: This thematic review suggests that this UK ambulance service is in a transition stage, with significant organisational, professional, and cultural challenges. The dichotomies, boundaries, and development issues are part of the development of an emerging profession for which it is essential that the educational agenda is addressed.

A mbulance services across the UK are increasingly aware of the need to evaluate their educational provision and to consider new and innovative operational practice. ${ }^{1}$ Government ministers have been emphasising the importance of team working, maximising the contribution of all staff to patient care, modernising and advancing education and training, and expanding the workforce. $^{2-4}$ Pressure is being applied to all those involved in health care education to produce personnel who are "fit for practice" within a seamless service and to break down what Milburn calls "tribalism", ${ }^{56}$ reducing the barriers between professions and specialities.

Recent work by Williams ${ }^{7}$ indicates that the current system of paramedic training concentrates on life threatening conditions with protocol driven practice, based on limited underpinning knowledge. The study highlights the need for work based and vocational learning. Others have raised issues concerning the need for a move from surface to deep thinking strategies, a greater decision making repertoire, and, for example, "a greater emphasis on deciding whether or not to treat patients before transporting to hospital", 8 an issue raised in a report on the need for advanced emergency care practitioners (ECPs), ${ }^{9}$ specialist paramedics educated to degree or masters level with the skills and depth of understanding for advanced autonomous practice.

Currently there are four levels of practitioner in most UK ambulance trusts. Ambulance care assistants (ACAs) undertake non-urgent patient transport work and are trained in first aid and driving skills over a few weeks. Ambulance technicians work in the accident and emergency (A\&E) field and are trained in advanced driving techniques and advanced first aid, including defibrillation and administration of some drugs (not intravenous). Their training runs for approximately 3-4 months with 12 months' probation. This is the most common entry level into the ambulance service, although a number of staff progress from ACA. Currently, only qualified technicians, with 12 months' experience following their probation, can access a paramedic course. Courses tend to run over 10 weeks, six of which are classroom based and four in hospital placements (usually A\&E, operating theatres, and obstetrics). Courses cover anatomy and physiology, and a large range of drugs and skills such as intravenous cannulation and intubation. There is, however, some regional variation, and a few universities have started to run direct entry graduate level programmes for paramedics. The fourth level practitioner is the aforementioned graduate ECP, of which there are only a few in the UK. In the south west of England, the Westcountry Ambulance NHS Trust (WAST) and the local hospital trusts have supported, for the last 4 years, the first multiprofessional (nurses and paramedics) BSc in Emergency Care, run at the University of Plymouth. The first seven paramedic ECPs are now in practicee, with early reports indicating that their educational development has been worthwhile. ${ }^{10}$

In the UK, the Institute for Healthcare Development (IHCD) is the awarding body for ACA, technician, and paramedic qualifications. IHCD is a department of Edexcel, which is the largest UK awarding body, offering a range of general and specialist qualifications. IHCD prescribe the curriculum, content, and assessments for all ambulance training throughout the UK.

Paramedics have recently (in 2003) gained professional registration with the newly formed Health Professions Council (HPC), which lists 12 professions allied to medicine, while the British Paramedic Association are working towards a professional body. ACAs and technicians are not professionally registered, although there are plans to include technicians on the register.

Abbreviations: $A \& E$, accident and emergency; $A C A$, ambulance care assistant; $E C P$, emergency care practitioner; IHCD, Institute for Healthcare Development; HE, higher education; HPC, Health Professions Council; NHS, National Health Service; WAST, Westcountry Ambulance NHS Trust 
Funding for training is quite different to all other professional groups in the National Health Service (NHS). Doctors, for example, are funded via the Department of Education and Skills, and nurses through NHS workforce development departments. By contrast, ambulance services have to fund training from their own exchequer budget, which inevitably creates direct conflict with operational requirements.

\section{METHODS}

The study was set in WAST in the south west of England, an ambulance trust that covers the counties of Cornwall, Devon, and Somerset and employs approximately 1300 staff, of which 1150 are clinical/operational. The majority of transported patients are conveyed to the six A\&E departments based within the region. The objective of the study was to develop an understanding of the current system and future development of training and education within WAST, based upon the experiences, beliefs and opinions of stakeholders (WAST and A\&E). The study was set up as a qualitative naturalistic inquiry ${ }^{11}$ using an illuminative evaluation approach $^{12}$ to capture stakeholders' perspectives by drawing upon constant comparisons within the data. The approach aimed to produce an evaluation that captured the dynamics of rapidly changing circumstances and environments to elicit a thematic review of training.

The sample was based on a stratified purposive sample with the objective of reaching data saturation within the key themes and proportional representation of each of the main grades of staff (table 1 ).

Data werS collected through audiotaped, semi-structured individual interviews. On two occasions, respondents were paired. Five focus group interviews were performed and were designed to promote interaction within the group to stimulate individual views on training and education. Two trained interviewers were involved, dividing up the interviews, which lasted for approximately 30 minutes.

Interviews focused on how respondents had been trained, what their views were of the current system of training and education, and how the system could be improved. The investigators remained open to views and opinion, cautious about personal bias, but based on a broad understanding of the field for appropriate lines of questioning. Data was transcribed by the interviewers.

Data was analysed using the constant comparison method from which three steps were identified ${ }^{13}{ }^{14}$ for this study. Initially data was open coded with the naming and categorisation of data; it was then axial coded, whereby the open codes were drawn back together to make connections between categories, and finally selectively coded to produce,

\begin{tabular}{|llll|}
\hline \multicolumn{2}{|l|}{ Table 1 } & Sample of staff & \\
\hline $\begin{array}{lll}\text { Type of } \\
\text { interview }\end{array}$ & $\begin{array}{l}\text { Staff } \\
\text { group }\end{array}$ & Staff members & $\begin{array}{l}\text { No of } \\
\text { people } \\
\text { interviewed }\end{array}$ \\
\hline Individual & WAST & $\begin{array}{l}\text { Paramedics } \\
\text { Technicians }\end{array}$ & 11 \\
& & ACAs & 7 \\
& & Training officers & 5 \\
Paired & & Managers & 1 \\
interviews & & ACAs & 2 \\
Focus group & & Emergency care & 1 \\
& & practitioners $(\times 4)$ & \\
Stakeholders & Other & A\&E consultants & 4 \\
& NHS & A\&E managers & 6 \\
Interview total & & A\&E nurses & 3 \\
\hline & & & 44 \\
\hline
\end{tabular}

from a more abstract level of analysis, the key emergent theme and model. The filed notes and transcripts were coded and concepts compared with the aid of the software program QSR N5.

\section{RESULTS}

Data collection ran from September 2002 to September 2003. A total of 44 interviews were performed, comprising 61 individuals ( 43 men, 18 women). There were 26 individual interviews, two paired interviews, five focus group interviews (four individuals in each group), and 11 stakeholder interviews, representing each of the six regional A\&E departments. Table 1 shows the interview breakdown. The WAST respondents' experience within the ambulance service ranged from 1 to 26 years (mean 7.9).

Each of the key themes is listed below, supported by extracts from the data. Data have been condensed for publication; full transcripts are available on request. Each quotation is referenced to the original source, for example FG-1(2) refers to focus group 1 page 2 of the record, I-14(4) refers to an individual interview, and St-2(3) to an A\&E stakeholder.

\section{Curriculum issues (basic training) \\ Entry level (accrediting prior learning)}

The current entry level set by most UK ambulance trusts to a technician programme is four GCSEs at A-C grade. While respondents accepted that there was a need for academic credibility, they challenged the notion that this should be measured in GCSEs. They argued that there were other equivalents, both experiential and certificated, and that entrance exams should be available and relevant, for example:"....just because they didn't work (at school) when they were 16 doesn't mean they're stupid at 35. I mean you can do an entrance exam to Oxford so why have you got to have O-levels to get into the ambulance service?" (I-16(2)).

\section{Programme issues (content and time)}

General views on the current system of training indicated that there was a high standard of teaching, with a much improved teaching environment. Training staff demonstrated an understanding of learning styles and the need to adapt their techniques for the learner.

However, key themes emerged around the "focus of training". Respondents trained prior to the 1990s tended to cite the benefits of earlier programmes as a focus on skills with more time available. This is probably because the demands and expectations on ambulance service personnel were significantly lower 10 or 20 years ago, yet despite significant advances in pre-hospital care much the same time is allocated now, producing what one stakeholder referred to as a "sausage factory" (St-7(1)).

A\&E stakeholders emphasised the need for a comprehensive skills development programme, with updates, taught in skills centres, not on patients. Concerns were raised over the "lack of hands on with real people" (I-2(1)) and respondents also challenged the balance between skill and academic development and the content relevance of the courses "... too many academic demands within the current time scale" (FG$3(2))$. Within the given time constraints they pushed for a focus on skills and on their perception of the "need to know": "OK so they know what the structure of the blood is but how is that going to improve their clinical skills on the road?" (I-2(3)).

Block courses and the dispersed nature of ambulance trusts demands that most courses are residential. This has a significant impact on individuals' working lives and their educational development. Some individuals preferred this approach to learning as it enabled them to concentrate and 
focus, but others were very unhappy about the displacement from their families and the "cramming" nature of the courses.

\section{Assessment and competency}

IHCD produce multiple choice questionnaires, short answer, and clinical skill assessment tools. These were seriously challenged by respondents for their errors of format and content relevance, "training to pass exams that we question" $(\mathrm{FG}-3(2))$, while there was a feeling that the process was used as a "tick box" exercise for the employers' legal liability.

In 2003, the ambulance service moved from clinical protocols to clinical guidelines produced by the Joint Royal Colleges Ambulance Liaison Committee. ${ }^{15}$ The underlying philosophy of this change highlights the need for ambulance staff to have a flexible approach and a deep insight into the variables influencing patient care. However, the A\&E stakeholders were concerned about the lack of a "competency framework" within the curriculum and how this could have been linked to the government's emerging salary framework, known as Agenda for Change.

\section{Moving to a graduate profession?}

The opinion was that paramedicine should be a graduate profession, with the need for credibility within the Health Professions Council (paramedics are one of the few nongraduate professions), and the need to develop questioning, evidence based practice moving from surface (rote learning) to deep thinking strategies, a point illustrated by a drug viva examiner who questioned students stock statements on "drug titration":

\section{"I would titrate it against the pain, they all said - they had learnt the phrase, they didn't understand the practice - and when I said to them "so you've got your $10 \mathrm{ml}$ of diluted Nubain $(20 \mathrm{mg})$ if you inject $4 \mathrm{ml}$, how much Nubain have you given?" none of them knew the answer" (St-7(2)).}

However, there were significant concerns about a move to the higher education (HE) sector: the need to make the work stimulating and interesting for graduates with a clear career ladder, and the possibility of workforce gaps in the lower grades as staff progress and concerns for those existing staff members who do not have the ability to make the move into HE.

\section{"I am not entirely sure that it is appropriate for everybody to be educated to degree level. I think the practical skills, common sense, the ability to get on with people are much more important" (St-7(2)).}

\section{Work based learning (mentorship and autonomous practice) Mentorship}

In the last few years there has been huge demands on ambulance services to meet response times, particularly for category A (acute/life threatening) calls where $75 \%$ of calls must be attended in 8 minutes. These demands have necessitated significant recruitment drives, with large influxes of trainees. Training staff have been heavily committed to these programmes and other support staff members have been allocated clinical duties with a subsequent lack of support for work based learning.

"I was left to my own devices, feeling vulnerable, with no one to turn to" (1-1(3)).

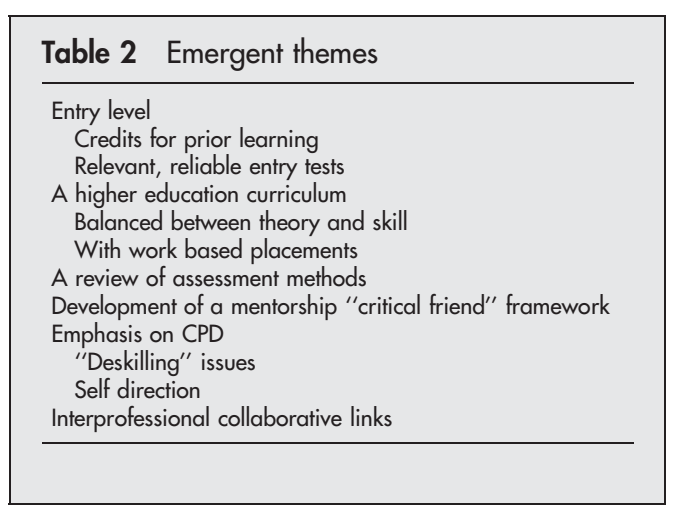

These issues are heightened by physical constraints to the mentorship role. In ambulances, closed bulkhead doors have been introduced as a security measure, which isolates trainees from their colleagues.

To gain experience, students are encouraged to "ride out" in ambulances; as they are normally manned by two members of staff, this experience is called third manning. The experience is greatly valued by trainees and other staff, but has been cut to the minimum as other pressures come to bear. Typical responses were: "The third manning I did taught me much more than anything else" (I-9(1)), and from the training staff: "You say to them, tell me about yourself and they say "Oh, I worked in insurance before". You say have you had any contact with patients, they say "No". You say have you seen anyone who has been injured, "No". Here we are, after they've done their training, putting them on the front line" (FG-1(2)).

Unlike many other healthcare professionals, ambulance staff tend to work alone or in pairs, in situations where their practice is rarely challenged. This highlights the need for adequate mentorship and a "critical friend", a point highlighted by one of the A\&E stakeholders: "You are accountable for everything that you do, but if you don't know the limit of your competence how do you know that you are doing something safely?" (St-6(2)).

Many respondents demonstrated an insight into the reasons for the current failings within the system and highlighted the issues of poor government funding and a target driven NHS. For example, one of the A\&E stakeholders pointed out: "I think unfortunately targets have taken over the world and the pursuit of targets is sometimes done to the detriment of the long term strategy" (St-7(2)).

\section{Continuous professional development}

Respondents expressed concerns over deskilling and the training for skills that were rarely used. The population and geographical diversity of the region and the pressure for response times has led to long periods of "standby" for staff (for example, in a layby at the edge of the road) and to a reduction in expertise in specific conditions: "I went to a road traffic accident with a paramedic ... who asked me to take the lead because he hadn't been to a road traffic accident in over a year" (I-2(3)). However, the A\&E stakeholders were impressed by the level of skills and were quick to point out the difficulty of their working conditions.

IHCD require paramedics to attend annual updates and a recertification programme at least every five years. The courses were generally well received, especially where they focused on infrequently practiced skills. Part of the recertification process includes a hospital placement; there were mixed views on the value of such practice, which depended on students' proactivity and the availability of patients. A\&E departments were also concerned about the number of students and the availability of support. However, they were 
universal in their acknowledgment that it was a valuable process for creating multi-professional links, breaking down barriers and enhancing communication: “...we can give them what isn't written down at all and that is what they learn about the social structure of the accident and emergency department" (St-3(1)), and from a paramedic respondent: "We need to be more integrated with other medical professions, we need to know more about their roles and skills" (I-10(3)).

\section{DISCUSSION}

This study is set at a time of change for paramedic education. There is a lack of consensus over paramedic roles, the advantages of advanced skills, and the need to respond to all cases, ${ }^{1617}$ but there is agreement that the role should be extended, with a requirement for an educational programme that supports the need for flexible guidelines as opposed to rigid protocols. ${ }^{18}$ With these issues in mind, the Open University and UNISON, a public sector trade union, are consulting stakeholders on the development of a national foundation degree. The Department of Health's "Changing Workforce" programme is supporting an ECP pilot programme at Warwick University, and groups such as the Higher Education Ambulance Development Group are developing competency frameworks.

This study is small, but is one in which key themes emerged, which is not inconsistent with qualitative work, in which "saturation" tends to be reached with a relatively small sample. Stratified purposive sampling, within the region, ensured that all the main grades were represented and thus should be a reasonable representation of views. While the results cannot be generalisable, it could also be argued that the work gives an indication of national opinion, as training is standardised by IHCD throughout the country and that working and government pressures in other Trusts are thus likely to be similar.

However, the reader should note that respondents had a tendency to emphasise the negative aspects of their working lives, despite interview questions focusing on the advantage of current systems of training and education. These results should therefore be taken as one aspect of a holistic review of staff roles, functions, and education within the existing constraints of the ambulance service, and therefore this report should not be considered in isolation. In addition, it is important to note that existing systems of training are largely constrained by the standards and guidelines of the IHCD, leaving little flexibility for local improvement initiatives.

To summarise the key themes (fig 1), concerns were raised about access to training programmes, and national entry levels of four GCSEs (soon to be increased to five) were not in themselves criticised, but there was vociferous support for accreditation of prior learning and the need for relevant reliable entry tests.

The current system of pre-qualification training does have significant advantages. It produces relatively skilled personnel in a short period of time, the standard of training is high, and the feedback from courses is good. Nevertheless, the view was that the curriculum did not reflect the requirements, standards, and practice of the workplace, a point supported by Kilner, ${ }^{19}$ who suggests that the curriculum plays little attention to the repertoire of skills and attributes demonstrated in other health professions and that "it is entirely possible that the curriculum is defining the attributes of the occupational group rather than the desirable attributes being used to define the curriculum" ${ }^{20}$

From this standpoint there was support for a move to HE, but with concerns over the balance between skill and theory, the practical and academic and "hands on" experience from early in the training. Such a move is likely to include the advantage of flexible access and flexible programmes of modular part time learning. The HE sector is also more likely to be able to offer a choice of pathways, without the necessity for block residential courses. In fact, services that offer only block residential courses could be considered discriminatory under British law, as women of childbearing age are significantly less likely to be able to attend. Fundamental to these changes are a source of external funding; it is essential that training and education is separated from the competing interests of operational requirements.

Assessment processes were criticised for their validity, reliability, and feasibility, with a clear need to identify alternative methods of assessment such as self and peer review and objective structured clinical examinations with links to a competency framework.

Major concerns were expressed about the availability of mentors and consequently patient safety, a situation that is emphasised by paramedics' autonomous practice. Respondents identified the government's target driven culture (in this case response times) as a contributor to the situation. In response to these views, WAST developed a mentorship programme in 2004.

Regarding contining professional development, the impression was that staff still expect the service to deliver their training; there was little self directed education and a resistance to invest in their own personal and professional development encapsulated by the comment "there is no education in the service, just training" (I-1(5)). Nevertheless there was an awareness of the need to change "...the importance of people taking ownership of their training" (I-2(4)). Concerns were raised over the deskilling of staff due to long periods of "standby", an issue that may be addressed with the development of regional skill centres, rotation of staff, and a refocus on the value of hospital placements.

Respondents were enthusiastic about the potential for interprofessional education, especially the collaborative benefits. However, the evidence for multiprofessional education is unclear, ${ }^{21-27}$ despite the government's drive for a core curriculum for doctors, nurses, and other healthcare professionals.

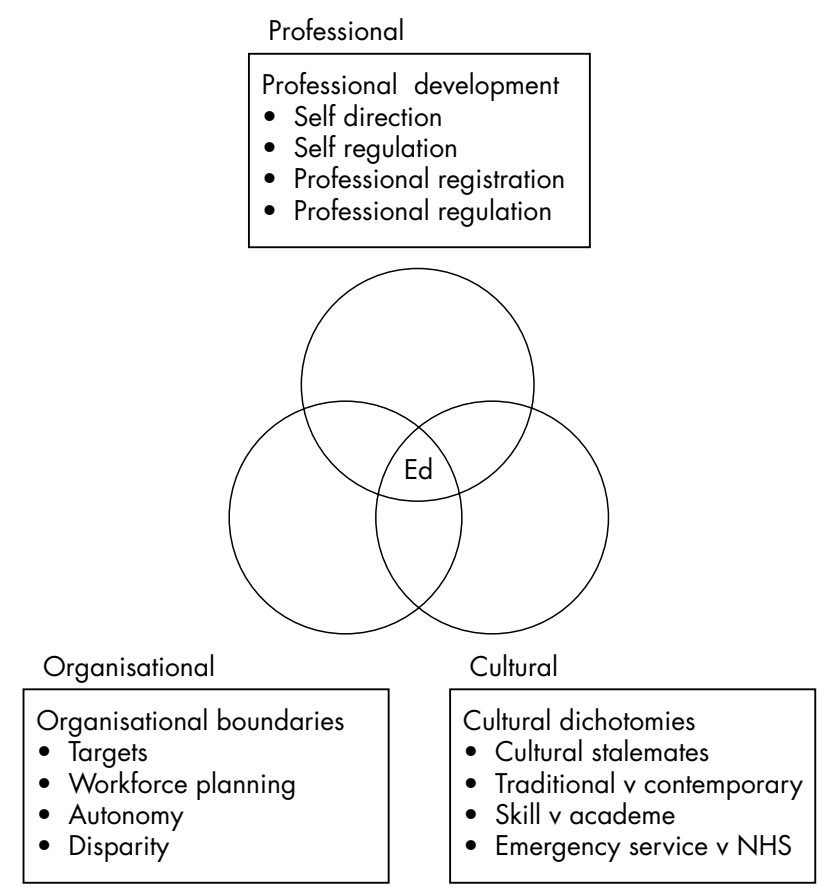

Figure 1 Educational drivers and suppressors. Ed, education. 
The methodological stance ${ }^{13}{ }^{14}$ taken in this paper included a third and final selective coding at a more abstract level to draw together the core categories. Fig 1 illustrates this as the contemporary education drivers and suppressors. The terms "training" and "education" have been used carefully throughout this paper, the former defined as a fixed process with a clearly defined beginning and end, the latter as an holistic continuous and ongoing development of the individual. Results from this study indicate that ambulance staff are in a transition between training and education.

There are significant cultural dichotomies, stalemates such as "they wouldn't give me time" versus "we won't give them time, they don't make the effort"; traditional (a patient transport service) versus contemporary views (autonomous self directed practice); the skill versus academe debate; and an emergency service or part of the NHS.

The organisational boundaries include: "target" agendas; workforce planning issues related to increasing demands from an ageing population; staff progression issues with the potential for workforce gaps the risks, threats, and advantages of increasingly self directed autonomous practice; and the disparity of work for a widely dispersed workforce in rural and urban settings.

Professional development includes issues of self direction and self regulation within the boundaries of safe practice, while recent HPC professional registration, without an as yet substantive regulatory body, are causing tensions within the profession.

These dichotomies, boundaries, and development issues are part of the development of an emerging profession for which it is essential that the educational agenda is addressed. It is recommended that policymakers consider such issues as entry level, the HE sector, and continuing professional development in relation to the emerging requirements of the profession.

\section{ACKNOWLEDGEMENTS}

Thanks to T Beer, Paramedic, Westcountry Ambulance Service NHS Trust for assistance with data collection.

Competing interests: none declared

\section{REFERENCES}

1 Battersby D. The future of ambulance officer education and training in the UK. Pre-hospital Immediate Care 1999;3:113-14.

2 Department of Health. A health service for all the talents: developing the NHS workforce. Consultation document on the review of workforce planning. London: Department of Health, 2000.
3 Department of Health. Reforming emergency care. London: Department of Health, 2000

4 Department of Health. Life in the fast lane. London: Department of Health, 1997.

5 Sanders C. Moves to get the NHS fighting fit. Times Higher Educ Suppl 8 June, 2001.

6 Bulstrode C, Bell Y, Gray M. Senior house officers: the lost tribes. Br J Hosp Med 1993;50:572-3.

7 Williams N. PEC Development, from concept to reality. London: Joint Royal Colleges Ambulance Liaison Committee Annual Conference, 2002.

8 Ambulance Service Association \& Medical Care Research Unit. The future of the ambulance service in the United Kingdom. Ambulance Service Association \& Medical Care Research Unit, 2001.

9 Joint Royal Colleges Ambulance Liaison Committee. The future role and education of paramedic ambulance service personnel (emerging concepts). Joint Royal Colleges Ambulance Liaison Committee, 2000.

10 Cooper S, Barrett B, Black S, et al. The emerging role of the Emergency Care Practitioner. Emerg Med J 2004;21:614-18.

11 Erlandson D, Harris E, Skipper B, et al. Doing naturalistic enquiry. A guide to methods. Newbury Park: Sage, 1993.

12 Robson C. Real world research. Blackwell, Oxford UK, Cambridge USA, 1993:176.

13 Mertens D. Research methods in education and psychology. Integrating diversity with quantitative and qualitative approaches. Thousand Oaks, London, New Delhi: Sage Publications, 1998:352.

14 Strauss A, Corbin J. Basics of qualitative research. Newbury Park, CA: Sage, 1990.

15 JRCALC. Clinical practice guidelines, version 3, 2004. Available at: http:// www.warwickuniversity.nhs.uk.

16 Cooke M. Who needs paramedics in pre-hospital care? Pre-hosp Immed Care 1997; 1:50.

17 Johns B. Who needs paramedics in pre-hospital care? Pre-hosp Immed Care 1997; 1:50

18 Anonymous. Conclusions to the debate. Pre-hosp Immed Care 1997;1:50.

19 Kilner T. Educating the ambulance technician, paramedic, and clinical supervisor: using factor analysis to inform the curriculum. Emerg Med J 2004;21:379-85

20 Kilner T. Desirable attributes of the ambulance technician, paramedic, and clinical supervisor: findings from a Delphi study. Emerg Med J 2004;21:374-8.

21 Pirrie A. Rocky mountains and tired Indians: on territories and tribes. Reflections on multidisciplinary education in the health professions. $\mathrm{Br} \mathrm{Edu}$ Res J 1999;25:113-26.

22 Lockhart-Wood K. Collaboration between nurses and doctors in clinical practice. Br J Nurs 2000;9:276-80.

23 Risser D, Rice M, Salisbury M, et al. The potential for improved teamwork to reduce medical errors in the emergency department. Ann Emerg Med 1999;34:373-83.

24 Poulton BC, West MA. Effective multidisciplinary teamwork in primary healthcare. J Adv Nurs 1993;18:918-25.

25 Ummenhofer W, Amsler F, Sutter PW, et al. Team performance in the emergency room: assessment of inter-disciplinary attitudes. Resuscitation 2001;49:39-46.

26 Wilson T, Mires GJ. A comparison of performance by medical and midwifery students in multi-professional teaching. Med Ed 2000;34:744-6.

27 Barr H, Hammick M, Koppel I, et al. Evaluating inter-professional education: two systematic reviews for health and social care Br Educ Res J $1999 ; 25: 533-44$ 